\title{
Discussion/Reply
}

\section{Discussion on "Review of Physical and Numerical Approaches for the Study of Gas Stirring in Ladle Metallurgy"}

\section{DIPAK MAZUMDAR and R.I.L. GUTHRIE}

https://doi.org/10.1007/s11663-019-01754-2

(C) The Minerals, Metals \& Materials Society and ASM International 2019

Physical and mathematical modeling of ladle metallurgy steelmaking has been a subject of great interest to steelmakers for several decades. During the last fifty years or so, numerous investigations have been carried out, both in academia and in industry, to help create a robust knowledge base for ladle metallurgy steelmaking. Parallel to these, excellent review articles have also been published by several investigators summarizing periodic progresses. ${ }^{[1-4]}$ The first review on the subject was published by the present authors ${ }^{[1]}$ in 1995 , to summarize the many physical and mathematical modeling studies on ladle metallurgy steelmaking carried out during the period 1976 to 1994 . This was followed by a subsequent review of the modeling of secondary steelmaking, by Sichen, ${ }^{[2]}$ almost twenty years later. A recent addition to these is the work of Liu and coworkers, ${ }^{[4]}$ in which, research work carried out during the last three decades, generally on physical modeling and numerical simulation of inert gas stirring in ladles, and particularly on (i) mixing, (ii) gas bubble formation, (iii) inclusion behavior, and (iv) open eye formation phenomena, has been summarized. The review ${ }^{[4]}$ is timely and summarizes a large number of investigations carried out in the above-mentioned areas, capturing successfully the progress in ladle metallurgy steelmaking research in recent years. Nonetheless, some obscurities, omissions, and errors remain in that work. ${ }^{[4]}$ These are pointed out in the following text where they are discussed further. They represent the purpose of the present communication.

DIPAK MAZUMDAR is with the Department of Materials Science and Engineering, Indian Institute of Technology, Kanpur 2018016, India. Contact e-mail: dipak@iitk.ac.in R.I.L. GUTHRIE is with the McGill Metals Processing Centre, McGill University, Montreal, QC, Canada.

Manuscript submitted September 14, 2019.

Article published online December 18, 2019.
Thus, in recent years, multiphase (gas-metal or gas-metal and slag) flow modeling of gas-stirred ladle systems has been widespread. Accordingly, a great deal of information on the subject is now documented in the literature. In their recent review, different approaches used by researchers to model two- and three-phase flows, plus associated governing equations, etc., were considered lucidly and summarized systematically ${ }^{[4]}$ with reference to four principal flow modeling approaches. They were (i) quasi-single-phase approximations, (ii) VOF (volume of fluid), (iii) Euler-Euler (E-E), and (iv) Eulerian-Lagrangian (E-L), including VOF. While the compilation is fairly comprehensive, ${ }^{[4]}$ any critical assessment and/or discussion of flow modeling approaches has been largely avoided by the authors. ${ }^{[4]}$ For example, Liu and coworkers ${ }^{[4]}$ considered Figure 1 to be sufficiently conclusive to suggest that the Euler-Euler (E-E) model is best suited to numerically simulate flow phenomena in argon-stirred ladles. Such a supposition, as discussed below, can be contested on the basis of experimental and computational results (also included in the review ${ }^{[4]}$, based on the flow modeling study (PBM coupled with E-E approach) by Li et al., ${ }^{[5]}$ presented in Figures 2(a) through (c). These clearly indicate that bubble diameter, gas volume fraction and mixing time, etc., in a three-phase, water-model ladle system can be predicted with equal, or superior, accuracy via an $\mathrm{E}-\mathrm{E}$ hydrodynamic model embodying bubble population balance (PBM) in conjunction with the $k-\varepsilon$ turbulence model. ${ }^{[5]}$ Toward these, it is important to mention here that currently available hydrodynamic models of gas-stirred ladle systems rely on many assumptions, including constant bubble size (as in the E-L approach), binary bubble collisions and breakage (as in PBM), negligible thermal interactions between gas and liquid, and so on.

As research work in this area continues, it is perhaps premature to side with a particular class of flow modeling procedures, given that exhaustive comparisons between industrial/pilot scale operations and numerical predictions are still largely pending. Indeed, depending on the phenomena of interest, even a very simple flow modeling approach can work satisfactorily. For example, if the primary goal of numerical computations is to predict bulk liquid mixing rates in an inert gas-stirred ladle, the quasi-single-phase procedure is known to be equally effective as the more elaborate, two-phase flow modeling approaches. ${ }^{[1]}$

Following their summary of a large number of studies on two- and three-phase modeling of gas-stirred ladle systems, Liu et al. ${ }^{[4]}$ consolidated their review and proposed a schematic to suggest various preferred combinations of numerical models for simulation of different phenomena in different regions of a gas-stirred 
ladle system. Reproduced from Reference 4, this is shown as Figure 3 in the present response. There, with reference to the modeling of slag entrapment and inclusion behavior at the slag-metal interface, the authors $^{[4]}$ have suggested a mathematical modeling formalism based on the VOF + LES approach. For this, it is instructive to note that classical VOF accommodates a surface tension force as the sole mechanism of momentum exchange between participating phases in any system. Since flow, as well as large-scale slag-metal interactions and slag entrainment phenomena in gasstirred ladles, is known to be caused by the bubbleinduced drag forces, it follows that the computation of drag force and the bubble-driven flow fields are prerequisites to the modeling of slag-metal interactions in such

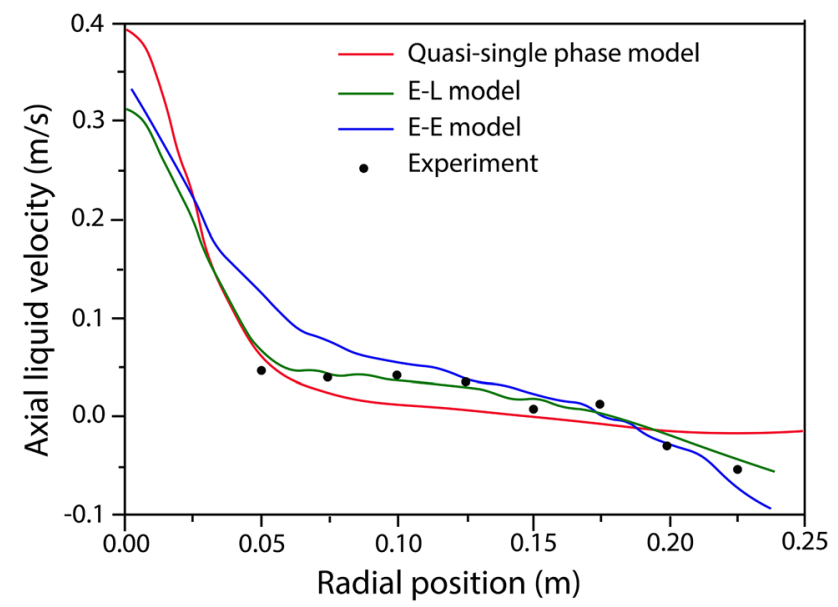

Fig. 1-Comparison of predicted and measured axial liquid velocities at $L / H=0.5$ from the bottom, illustrating a performance comparison of three different flow modeling approaches (reprinted from Ref. [4]). systems. Given this, it is apparent that unless the momentum conservation equation in the VOF procedure is appropriately amended, and bubble-induced drag force (see later) is accommodated explicitly, the effect of convection currents induced by the upwelling plume or rising bubbles cannot be simulated realistically. Given the above, it is therefore reasonable to assume that the VOF + LES procedure is likely to prove inadequate to simulate a bubble-driven flow and associated phenomena in gas-stirred ladle systems. On the basis of these facts, the present authors submit that a "DPM + VOF (with an additional gas-liquid momentum exchange term) + the LES procedure" is likely to be more effective and more appropriate, to model slag entrainment phenomena in gas-stirred ladle systems. Indeed, a very similar approach, along the lines now suggested, has already been advanced ${ }^{[5]}$ and slag eye formation (owing to large-scale melt-slag-gas interactions) in such systems has been modeled reasonably effectively.

Referring to Figure 3, it is seen that two different, complimentary approaches have been advocated by Liu et al., for predicting de-S and mass transfer phenomenon from the first principles. Since slag-metal mass transfer was not included in the scope of their review, ${ }^{[4]}$ some discussion and analysis of this are therefore naturally warranted. Mass transfer coefficients, as well as slag-metal interfacial areas, as one would readily acknowledge, are the corner stone in the computation of melt-phase, transport-controlled, mass transfer processes. Recent work of Hoang et al. ${ }^{[6]}$ indicate that mass transfer coefficient can be estimated from the small eddy theory in terms of specific turbulence kinetic energy dissipation rate, $\varepsilon$, and the kinematic viscosity of the bulk liquid, $v$, according to:

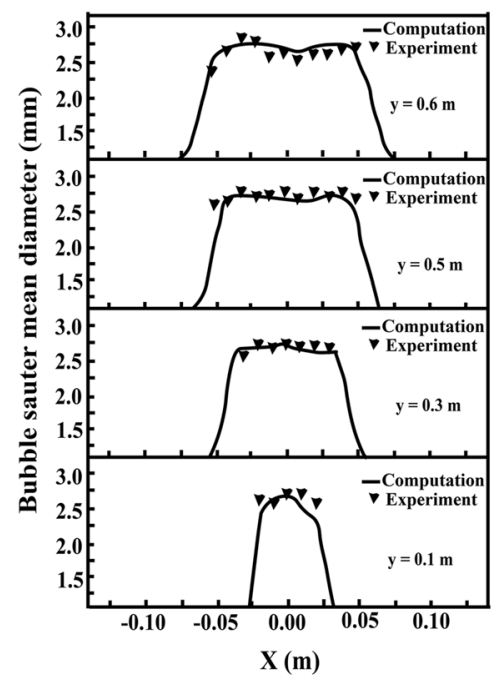

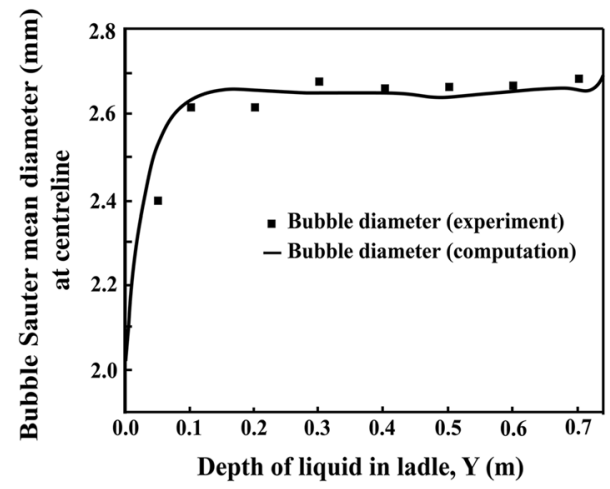

(b)

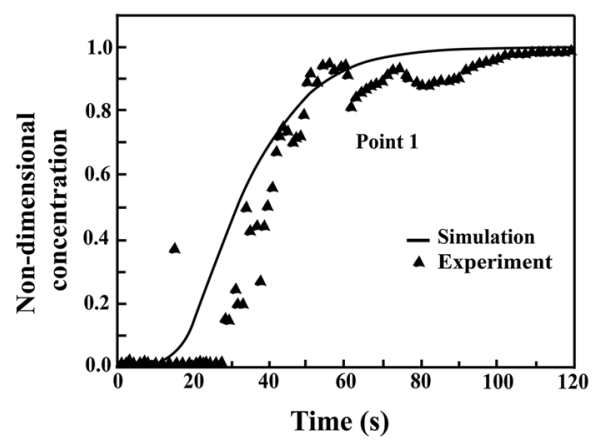

(c)

(a)

Fig. 2-Comparison between numerical prediction and experimental measurements, illustrating the adequacy of an E-E hydrodynamic model, coupled to a bubble population balance (PBM), and the $k-\varepsilon$ turbulence model. (a) Mean bubble diameter, (b) Sauter mean bubble diameter at centreline, and $(c)$ mixing time (From Li et al. ${ }^{[5]}$ with permission). 


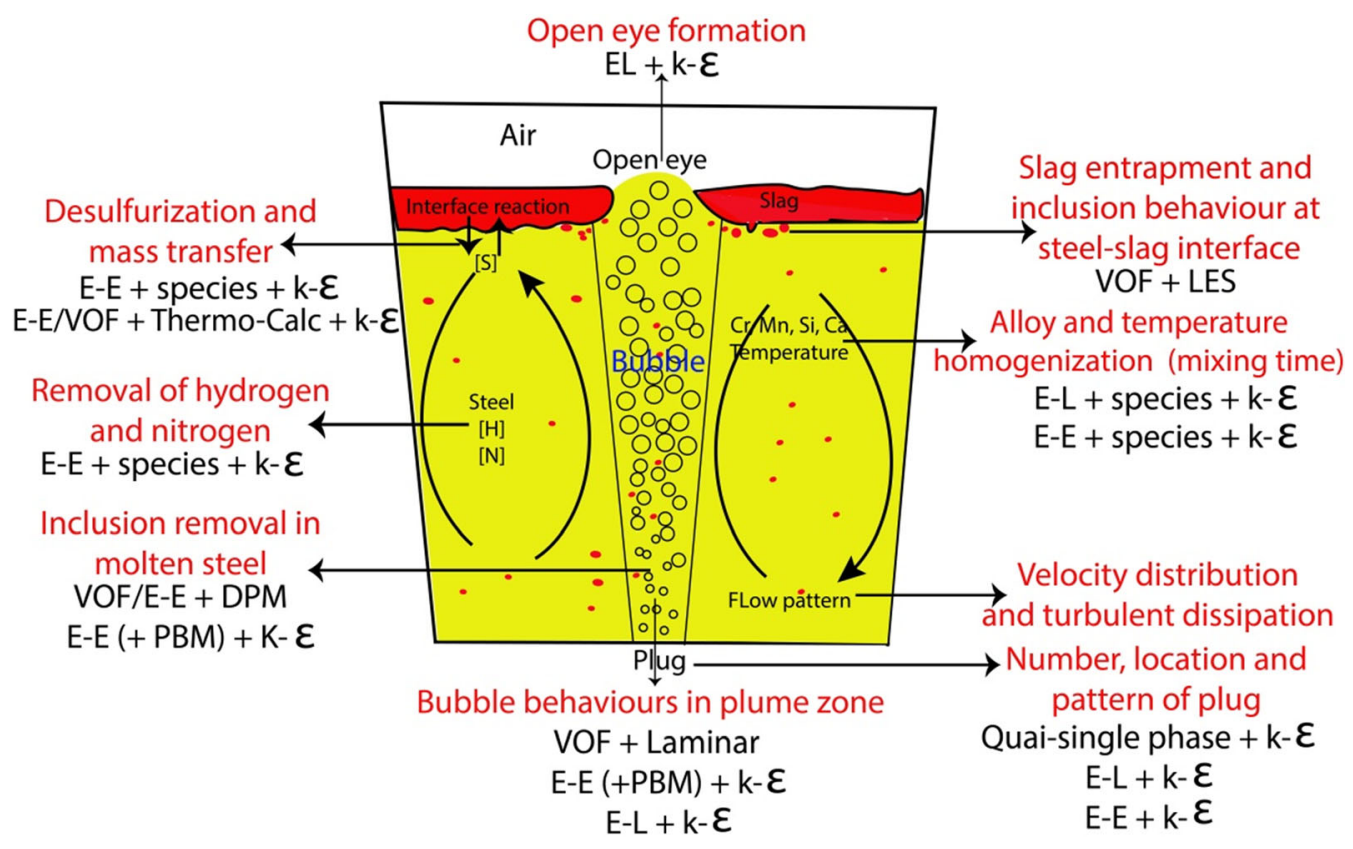

Fig. 3-A schematic of inert gas-stirred ladle system and various calculation schemes to investigate different phenomena (Reprinted from Ref. [4]).

$$
k=0.4 \sqrt{D}\left(\frac{\varepsilon}{v}\right)^{0.25}
$$

An appropriate turbulence model is therefore a prerequisite to the prediction of mass transfer coefficient in gas-stirred ladle systems. Mass transfer obeys first-order kinetics and the corresponding rate expression is conveniently represented as:

$$
\stackrel{\mathrm{o}}{m}=-\frac{\mathrm{d} C_{i}}{\mathrm{~d} t}=k\left(\frac{A_{\mathrm{int}}}{V}\right)\left(C_{i}-C_{i}^{e}\right)
$$

In Eq. [2], $C$ is the molar concentration $\left(\mathrm{kg} \mathrm{mol} / \mathrm{m}^{3}\right), i$ represents the transferring species, $\stackrel{\circ}{m}$ is the mass transfer rate $\left(\mathrm{kg} \mathrm{mol} / \mathrm{m}^{3} \mathrm{~s}\right), k$ is the mass transfer coefficient $(\mathrm{m} /$ $\mathrm{s}), A_{\text {int }}$ is the interfacial area $\left(\mathrm{m}^{2}\right)$, and $V$ is the volume of the melt $\left(\mathrm{m}^{3}\right)$. Evidently, an estimate of $A_{\text {int }}$ is needed in addition to Eq. [1], if the mass transfer rate is to be predicted via Eq. [2]. Toward this, the need for a multiphase flow modeling procedure, such as VOF + DPM or E-E, is readily apparent, as has been appropriately pointed out by Liu et al. It is, however, important to mention that in a vast majority of twofluid mass transfer studies of ladle metallurgy steelmaking, the estimation of slag-metal interfacial area has not been rigorous. Rather, it is generally deduced by considering an artificially enhanced, planar slag-metal interfacial area. In such a context therefore, the adequacy of two- or three-phase modeling procedures, such as the $\mathrm{VOF}+\mathrm{DPM}$ or the E-E, and various auxiliary models, is not known with any certainty. Clearly, more experimental work and plant scale measurements, as well as numerical simulations, are needed to substantiate such model study programs further. In addition to the above, the recommendation of a VOF + laminar flow model, as suggested in Figure 3 (see beneath the base of the ladle schematic) for investigating bubble behavior in the plume zone, does not appear to be sufficiently convincing and needs some clarification, since bubbles are known to exacerbate turbulence within an upwelling gas-liquid plume. ${ }^{[1]}$

Two- (melt-gas) and three-phase (melt-slag and gas) computations of gas-stirred ladle systems have been reported by many researchers in recent years and most of these have been referred to by Liu et al. ${ }^{[4]}$ (see, for example, Tables IX and X in Reference 4). There, the authors ${ }^{[4]}$ emphasized four different flow modeling approaches, i.e., (i) quasi-single-phase approximations, (ii) VOF (volume of fluid), (iii) Euler-Euler (E-E), and (iv) Eulerian-Lagrangian $(\mathrm{E}-\mathrm{L})$, giving practically no attention to the newly suggested, VOF + DPM flow calculation procedure ${ }^{[7]}$ A concise description and analysis of the combined $\mathrm{VOF}+\mathrm{DPM}$ approach is therefore presented here: the VOF and the DPM calculation procedures, as one will note here, rely on fundamentally different concepts. As such, their straight-forward coupling, as advocated by many investigators, may not be scientifically sound. To substantiate this observation, the governing equations of mixture and discrete-phase motions, in the coupled VOF + DPM formalism, represented in compact vector notation, ${ }^{[7]}$ are considered below;

Equation of mixture motion:

$$
\begin{aligned}
& \frac{\partial}{\partial t}\left(\rho_{\text {mix }} \bar{v}_{\text {mix }}\right)+\nabla \cdot\left(\rho_{\text {mix }} \bar{v}_{\text {mix }} \bar{v}_{\text {mix }}\right) \\
& \quad=-\nabla p+\nabla \cdot\left(\mu_{\mathrm{e}} \nabla \bar{v}_{\text {mix }}\right)+S_{v_{\text {mix }}}+\rho_{\text {mix }} \bar{g}+\bar{F}_{\mathrm{B}}+f_{\sigma}
\end{aligned}
$$


Equation of discrete-phase motion:

$$
\frac{\mathrm{d} \bar{V}_{b}}{\mathrm{~d} t}=\bar{F}_{\text {drag }}+\left(1-\frac{\rho_{1}}{\rho_{\mathrm{g}}}\right) \bar{g}
$$

The two preceding equations are then explicitly coupled via the drag force term $\bar{F}_{\text {drag }}$, defined as:

$$
\bar{F}_{\text {drag }}=\frac{3 \mu}{4 d_{\mathrm{b}}^{2} \rho_{\mathrm{g}}} C_{\mathrm{D}} \operatorname{Re}\left(\bar{V}_{\text {liq }}-\bar{V}_{\mathrm{b}}\right)
$$

Note that the drag force per unit volume of the liquid (represented as $\bar{F}_{\mathrm{B}}$ ), and the drag force per unit mass $\left(\bar{F}_{\text {drag }}\right)$, appearing in Eqs. [3] and [4] above, are interrelated. Furthermore, the Reynolds number, Re, appearing in Eq. [5] is expressed as:

$$
\operatorname{Re}=\frac{d_{\mathrm{b}} \rho_{\text {liq }}}{\mu}\left|\bar{V}_{\text {liq }}-\bar{V}_{\mathrm{b}}\right|
$$

Estimation of Re and $\bar{F}_{\text {drag }}$ (and hence, $\bar{F}_{\mathrm{B}}$ ), as seen from Eqs. [5] and [6], requires, from a rigorous stand point, the liquid velocity, $\bar{V}_{\text {Liq }}$, but this latter entity is not known explicitly from the flow equation (i.e., Eq. [3]), which is only specifically applicable to the gas-liquid mixture phase. As such, the VOF model is formulated on the basis of a single velocity scale ${ }^{[8]}$, i.e., the mixture velocity. Therefore, in the combined VOF + DPM calculation scheme, such as those employed in the metallurgical engineering literature, the Reynolds number and the drag force in the bubbly region $\left(\bar{F}_{\mathrm{B}}\right)$ can, at best, be estimated on the basis of a "mixture velocity." Incorporation of a "mixture velocity," in lieu of a liquid velocity, to estimate $\operatorname{Re}$ and $\bar{F}_{\mathrm{B}}$ is an ad hoc strategy and appears to be a serious conceptual limitation of the combined VOF + DPM formulations advocated recently in metallurgical engineering literature. Experimentally measured gas-liquid flows in water models are needed, as they could provide further insight and may justify, albeit not from a phenomenological stand point, the practicality of such approximations.

An understanding of gas-liquid coupling in ladle metallurgy operations is important and is central to the hydrodynamic modeling of such systems. It is rather well accepted ${ }^{[9]}$ that under ladle metallurgy steelmaking conditions, bubbles forming at nozzles or orifices rapidly devolve into a plume of widely dispersed spherical cap bubbles, leading to a gas voidage inside the upwelling plume in the range of 2 to 10 pct. Given the above, a depiction of densely populated, spherical bubbles in the upwelling gas-liquid mixture, such as the one shown in Figure 3, which may be adequate for a water-model ladle system, is quite incorrect and misleading. In addition to that, a centrally located porous plug, primarily a subject matter of academic interest, is rarely deployed in practice; off-centered porous plugs being far more typical of industrial operations. Finally, given that modern-day steel-processing ladles are invariably physically covered, ambient air within the entire

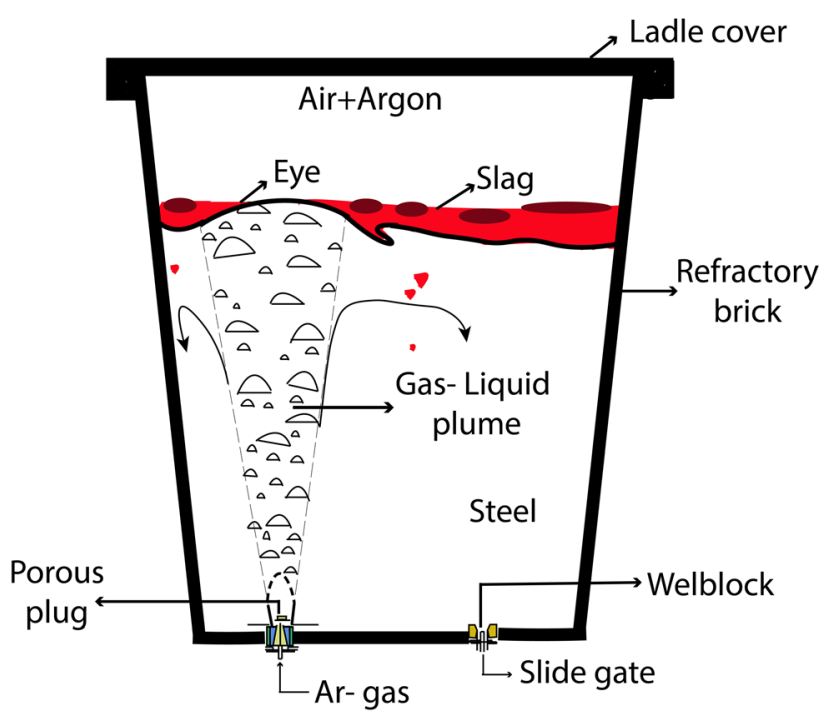

Fig. 4-Authors' impression of ladle stirring and a modern-day ladle-refining furnace (LRF).

ladle free board volume, as suggested through Figure 3, can hardly be justified. The present authors' impression of ladle stirring and a ladle-refining furnace is depicted in Figure 4.

The three major reviews, published during the last three decades and a half, confirm significant progress on physical and mathematical modeling of ladle metallurgy steelmaking to show that sufficiently advanced mathematical models have been formulated and their implications worked out successfully. Despite such advances, modeling of non-isothermal, reacting, multiphase flow phenomena, which are of paramount importance in actual industrial practice, have been few and far between. It is therefore still not known with any certainty, to what extent different classes of mathematical models perform in real-life process simulations. While efforts must continue to include more complex physics into existing models and thereby enhance their predictive capabilities further, the need for parallel water modeling, as well as high temperature field trials, should not be neglected, or undermined. Widespread application and validation of mathematical models on the shop floor are the need of the hour, so one can make a pragmatic assessment of the various advances in the published literature.

So, as a final point, the archival importance of the review by Liu and coworkers, ${ }^{[4]}$ according to the present authors, could have been increased substantially, had a brief summary of practical ladle metallurgy steelmaking been included as a preamble. Similarly, a concise and critical account of notable previous contributions on such issues as gas-liquid coupling, bubble distribution and voidage, long- and short-term wandering of the plume, turbulence production by bubbles, etc., would have helped in maintaining continuity with previous reviews. It is also equally desirable that an authoritative review, beyond compiling the state of the art, also points out various gaps in the literature and the preferred directions along which future research ought to move. 
These suggestions are advanced, not as criticisms, but to improve the articulation of future review articles, so as to render them more comprehensive and effective.

\section{REFERENCES}

1. D. Mazumdar and R.I.L. Guthrie: ISIJ Int., 1995, vol. 35, pp. 1-20.

2. L. Zhang and S. Taniguchi: Int. Mater. Rev., 2000, vol. 45, pp. 59 82.

3. D. Sichen: Steel Res. Int., 2012, vol. 83, pp. 825-41.

4. Y. Liu, M. Errson, H. Liu, P.G. Jonson, and Y. Gan: Metall. Mater. Trans. B, 2019, vol. 50B, pp. 555-77.
5. L. Li, Z. Liu, B. Li, H. Matsuura, and F. Tsukhashi: ISIJ Int., 2015, vol. 55 (7), pp. 1337-45.

6. Q.N. Hoang, M.A. Ramirez-Argaez, A.H. Conejo, B. Blanpain, and A. Dutta: JOM, 2018, vol. 70, pp. 2109-18.

7. G. Chen, S. He, and Y. Li: Metall. Mater. Trans. B, 2017, vol. 48B, pp. $2176-88$.

8. D. Mazumdar and J.W. Evans: Modelling of Steelmaking Process, CRC Press, Boca Raton, 2009.

9. Y. Sahai and R.I.L. Guthrie: Metall Trans B, 1982, vol. 13B, pp. 203-11.

Publisher's Note Springer Nature remains neutral with regard to jurisdictional claims in published maps and institutional affiliations. 